\title{
Robust Reliable Stabilization of Switched Nonlinear Systems with Time-Varying Delays and Delayed Switching
}

\author{
Zhengrong Xiang and Qingwei Chen \\ School of Automation, Nanjing University of Science and Technology, Nanjing 210094, China \\ Correspondence should be addressed to Zhengrong Xiang, xiangzr@mail.njust.edu.cn
}

Received 29 September 2009; Revised 18 April 2010; Accepted 11 May 2010

Academic Editor: John Burns

Copyright (c) 2010 Z. Xiang and Q. Chen. This is an open access article distributed under the Creative Commons Attribution License, which permits unrestricted use, distribution, and reproduction in any medium, provided the original work is properly cited.

This paper is concerned with the problem of robust reliable stabilization of switched nonlinear systems with time-varying delays and delayed switching is investigated. The parameter uncertainties are allowed to be norm-bounded. The switching instants of the controller experience delays with respect to those of the system. The purpose of this problem is to design a reliable state feedback controller such that, for all admissible parameter uncertainties and actuator failure, the system state of the closed-loop system is exponentially stable. We show that the addressed problem can be solved by means of algebraic matrix inequalities. The explicit expression of the desired robust controllers is derived in terms of linear matrix inequalities (LMIs).

\section{Introduction}

A switched system is composed of a family of continuous-time or discrete-time subsystems and a rule specifying the switching among them. Switched systems have received increasing attentions in the past few years, since many real-word systems such as mechanical systems, automotive industry, aircraft, and air traffic control systems, chemical processes can be modelled as switched systems (see [1-3]). A large number of results have been reported for such systems (see [4-8]).

On the other hand, time delay systems have continuously been receiveing considerable attention over the past decades. The main reason is that many kinds of engineering systems, for instance, long-distance transportation systems, hydraulic pressure systems, network control systems, and so on, include time delay phenomena in their dynamics. Many valuable results have been obtained for switched systems with time delay (see [9-15]). On the other hand, the actuators may be subjected to failures in real practice, therefore, it is of practical 
importance to design a control system which can tolerate faults of actuators. Several design approaches to the reliable controller have been proposed for linear and nonlinear systems (see [16-19]), and these results have been extended to switched systems (see [20-22]).

Recently, the asynchronous switching control problem of switched systems has stirred renewed research interests, and a varity of switched systems have been investigated by different approaches [23-26]. However, to the best of the authors' knowledge, the issue of reliable stabilization of switched nonlinear systems with time-varying delay under asynchronous switching has not been fully investigated, which motivated the present study.

In this paper, we are interested in designing the robust reliable controller for uncertain switched nonlinear system with time-varying delays and delayed switching. The remainder of the paper is organized as follows. In Section 2, problem formulation is presented and the failure model of actuator in switched system is introduced briefly. In addition, some necessary lemmas are given. In Section 3, based on the average dwell-time approach, controller design for switched nonlinear system with time-varying delays and delayed switching is developed, and sufficient conditions for the existence of the controller are formulated in terms of a set of matrix inequalities. Concluding remarks are given in Section 4.

Notation. Throughout this paper, the superscript " $T$ " denotes the transpose, $\|\cdot\|$ denotes the Euclidean norm. $\lambda_{\max }(P)$ and $\lambda_{\min }(P)$ denote the maximum and minimum eigenvalues of matrix $P$, respectively, $I$ is an identity matrix with appropriate dimension. $\operatorname{diag}\left\{a_{i}\right\}$ denotes diagonal matrix with the diagonal elements $a_{i}, i=1,2, \ldots, n$. The asterisk $*$ in a matrix is used to denote term that is induced by symmetry. The set of positive integers is represented by $Z^{+}$.

\section{Problem Formulation and Preliminaries}

Consider the following uncertain nonlinear switched system with actuator fault

$$
\begin{gathered}
\dot{x}(t)=\widehat{A}_{\sigma(t)} x(t)+\widehat{A}_{d \sigma(t)} x\left(t-d_{\sigma(t)}(t)\right)+B_{\sigma(t)} u^{f}(t)+f_{\sigma(t)}(x(t), t), \\
x(t)=\varphi(t), \quad t \in\left[t_{0}-d, t_{0}\right],
\end{gathered}
$$

where $x(t) \in R^{n}$ is the state vector, $u^{f}(t) \in R^{l}$ is the control input of actuator fault, $\varphi(t)$ is a continuous vector-valued function. The function $\sigma(t):\left[t_{0}, \infty\right) \rightarrow \underline{N}=\{1,2, \ldots, N\}$ is the switching signal which is deterministic, piecewise constant and right continuous, that is, $\sigma:\left\{\left(t_{0}, \sigma\left(t_{0}\right)\right),\left(t_{1}, \sigma\left(t_{1}\right)\right), \ldots\right\}, k \in Z^{+}$, where $t_{0} \geq 0$ is the initial time, and $t_{k}$ denotes the $k$ th switching instant. $d_{\sigma(t)}(t)$ denotes the time-varying state delay satisfying $0<d_{\sigma(t)}(t) \leq$ $d, \dot{d}_{\sigma(t)}(t) \leq \tau$ for constants $d$ and $\tau$. Moreover, $\sigma(t)=i$ means that the $i$ th subsystem is activated. $N$ denotes the number of subsystems. $f_{i}(x(t), t)(i \in \underline{N})$ are nonlinear functions satisfying

$$
\left\|f_{i}(x(t), t)\right\| \leq\left\|U_{i} x(t)\right\|,
$$

where $U_{i}$ are known real constant matrices. 
$\widehat{A}_{i}, \widehat{A}_{d i}$ for $i \in \underline{N}$ are uncertain real-valued matrices with appropriate dimensions, and have the following form:

$$
\left[\begin{array}{ll}
\widehat{A}_{i} & \widehat{A}_{d i}
\end{array}\right]=\left[\begin{array}{ll}
A_{i} & A_{d i}
\end{array}\right]+H_{i} F_{i}(t)\left[\begin{array}{ll}
E_{1 i} & E_{2 i}
\end{array}\right]
$$

where $A_{i}, A_{d i}, B_{i}, H_{1 i}, E_{1 i}, E_{2 i}$ are known real constant matrices with proper dimensions, and $H_{1 i}, E_{1 i}, E_{2 i}$ denote the structure of the uncertainties, $F_{i}(t)$ are unknown time-varying matrices which satisfy

$$
F_{i}^{T}(t) F_{i}(t) \leq I
$$

The control input of actuator fault $u^{f}(t)$ can be described as

$$
u^{f}(t)=M_{\sigma(t)} u(t)
$$

where $u(t)=K_{\sigma(t)} x(t)$ is the switching controller which will be designed, $M_{i}(i \in \underline{N})$ are the actuator fault matrices with the following form:

$$
M_{i}=\operatorname{diag}\left\{m_{i 1}, m_{i 2}, \ldots, m_{i l}\right\}, \quad 0 \leq \underline{m}_{i k} \leq m_{i k} \leq \bar{m}_{i k}, \bar{m}_{i k} \geq 1, k=1,2, \ldots, l .
$$

For simplicity, we introduce the following notation

$$
M_{i 0}=\operatorname{diag}\left\{\tilde{m}_{i 1}, \tilde{m}_{i 2}, \ldots, \tilde{m}_{i l}\right\}, \quad J_{i}=\operatorname{diag}\left\{j_{i 1}, j_{i 2}, \ldots, j_{i l}\right\}, \quad L_{i}=\operatorname{diag}\left\{l_{i 1}, l_{i 2}, \ldots, l_{i l}\right\},
$$

where $\tilde{m}_{i k}=1 / 2\left(\bar{m}_{i k}+\underline{m}_{i k}\right), j_{i k}=\left(\bar{m}_{i k}-\underline{m}_{i k}\right) /\left(\bar{m}_{i k}+\underline{m}_{i k}\right), l_{i k}=\left(m_{i k}-\tilde{m}_{i k}\right) / \tilde{m}_{i k}$.

From (2.6)-(2.7), we have

$$
M_{i}=M_{i 0}\left(I+L_{i}\right), \quad\left|L_{i}\right| \leq J_{i} \leq I,
$$

where $\left|L_{i}\right|=\operatorname{diag}\left\{\left|l_{i 1}\right|,\left|l_{i 2}\right|, \ldots,\left|l_{i l}\right|\right\}$.

Remark 2.1. $m_{i k}=1$ means normal operation of the $k$ th actuator signal of the $i$ th subsystem. When $m_{i k}=0$, it covers the case of the complete failure of the $k$ th actuator signal of the $i$ th subsystem. When $\underline{m}_{i k}>0$ and $m_{i k} \neq 1$, it corresponds to the case of partial failure of the $k$ th actuator signal of the $i$ th subsystem.

The delayed switching of the controller can be shown in Figure 1.

We can see from Figure 1 that the controller $K_{i}$ operates the $i$ th subsystem in $\left[t_{k-1}+\right.$ $\left.\Delta_{k-1}, t_{k}\right)$, and operates the $j$ th subsystem in $\left[t_{k}, t_{k}+\Delta_{k}\right)$. 


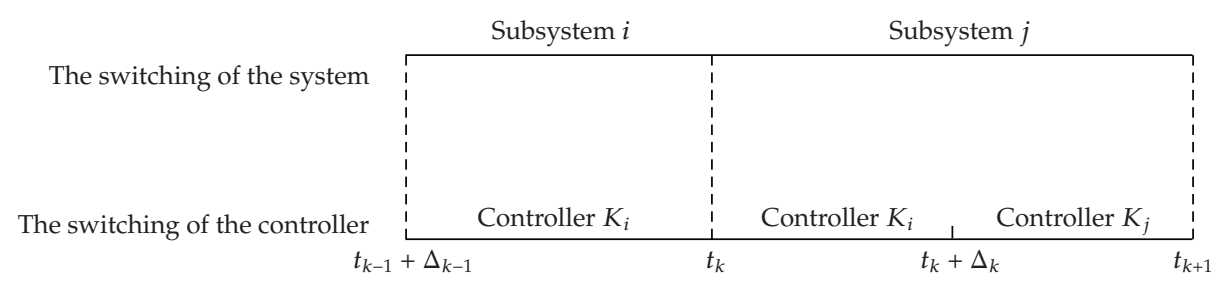

Figure 1: Diagram of the delayed switching.

Let $\sigma^{\prime}(t)$ denote the switching signal of the controller, the switching instants of the controller can be described as

$$
t_{1}+\Delta_{1}, t_{2}+\Delta_{2}, \ldots, t_{k}+\Delta_{k}, \ldots, k \in Z^{+}
$$

where $\Delta_{k}<\inf _{k \in Z^{+}}\left(t_{k+1}-t_{k}\right), \Delta_{k}$ represents the delayed period, and it is said to be mismatched period.

Remark 2.2. Mismatched period $\Delta_{k}<\inf _{k \in Z^{+}}\left(t_{k+1}-t_{k}\right)$ guarantees that there always exists a period that the controller and the system operate synchronously, and this period is said to be matched period in the later section.

Due to the delayed switching, the real input of actuator fault can be written as

$$
u^{f}(t)=M_{\sigma^{\prime}(t)} K_{\sigma^{\prime}(t)} x(t)
$$

Under switching controller (2.10), the resulting closed-loop system is given by

$$
\begin{gathered}
\dot{x}(t)=\left(A_{\sigma(t)}+B_{\sigma(t)} M_{\sigma^{\prime}(t)} K_{\sigma^{\prime}(t)}\right) x(t)+A_{d \sigma(t)} x\left(t-d_{\sigma(t)}(t)\right)+f_{\sigma(t)}(x(t), t), \\
x(t)=\varphi(t), \quad t \in\left[t_{0}-d, t_{0}\right] .
\end{gathered}
$$

System (2.1) without uncertainties and actuator fault can be written as

$$
\begin{gathered}
\dot{x}(t)=A_{\sigma(t)} x(t)+A_{d \sigma(t)} x\left(t-d_{\sigma(t)}(t)\right)+B_{\sigma(t)} u(t)+f_{\sigma(t)}(x(t), t), \\
x(t)=\varphi(t), \quad t \in\left[t_{0}-d, t_{0}\right] .
\end{gathered}
$$

Definition 2.3 (see [13]). If there exists switching signal $\sigma(t)$, such that the trajectory of system (2.1) satisfies $\|x(t)\| \leq \alpha\left\|x\left(t_{0}\right)\right\|_{h} e^{-\beta\left(t-t_{0}\right)}$, then system (2.1) is said to be exponentially stable with convergent rate $\beta$, where $\alpha \geq 1, \beta>0, t \geq t_{0},\|x(t)\|_{h}=\sup _{-d \leq \theta \leq 0}\{\|x(t+\theta)\|,\|\dot{x}(t+\theta)\|\}$. 
Definition 2.4 (see [27]). For any $T_{2}>T_{1} \geq 0$, let $N_{\sigma}\left(T_{1}, T_{2}\right)$ denote the switching number of $\sigma(t)$ on an interval $\left(T_{1}, T_{2}\right)$. If

$$
N_{\sigma}\left(T_{1}, T_{2}\right) \leq N_{0}+\frac{T_{2}-T_{1}}{\tau_{a}}
$$

hold for given $N_{0} \geq 0, \tau_{a}>0$, then the constant $\tau_{a}$ is called the average dwell time and $N_{0}$ is the chatter bound.

The following lemmas play an important role in the later development.

Lemma 2.5 (see [28]). For given vectors $a, b$ and the positive matrix $X>0$, there exists the matrix $M$ with appropriate dimension, such that

$$
-2 a^{T} b \leq \inf _{X>0, M}\left\{\left[\begin{array}{l}
a \\
b
\end{array}\right]^{T}\left[\begin{array}{cc}
X & X M \\
M^{T} X & \left(M^{T} X+I\right) X^{-1}(X M+I)
\end{array}\right]\left[\begin{array}{l}
a \\
b
\end{array}\right]\right\} .
$$

Lemma 2.6 (see [29]). For matrices $X, Y$ of appropriate dimension and $Q>0$, we have

$$
X^{T} Y+Y^{T} X \leq X^{T} Q X+Y^{T} Q^{-1} Y
$$

Lemma 2.7 (see [30]). Let $U, V, W$, and $X$ be real matrices of appropriate dimensions with $X$ satisfying $X=X^{T}$, then for all $V^{T} V \leq I$,

$$
X+U V W+W^{T} V^{T} U^{T}<0
$$

if and only if there exists a scalar $\varepsilon>0$ such that

$$
X+\varepsilon U U^{T}+\varepsilon^{-1} W^{T} W<0 .
$$

Lemma 2.8 (see [31]). For matrices $R_{1}, R_{2}$ with appropriate dimension, there exists a positive scalar $\beta>0$, such that

$$
R_{1} \Sigma(t) R_{2}+R_{2}^{T} \Sigma^{T}(t) R_{1}^{T} \leq \beta R_{1} U R_{1}^{T}+\beta^{-1} R_{2}^{T} U R_{2}
$$

hold, where $\Sigma(t)$ is time-varying diagonal matrix, $U$ is known real-value matrix satisfying $|\Sigma(t)| \leq U$.

The objective of this paper is to design a reliable controller for system (2.1) with delayed switching such that the resulting closed-loop system is robust exponentially stable. 


\section{Main Results}

To obtain the main results of this paper, we first consider the stability of the following nonlinear delay system

$$
\begin{gathered}
\dot{x}(t)=A x(t)+A_{d} x(t-d(t))+f(x(t), t), \\
x(t)=\varphi(t), \quad t \in\left[t_{0}-d, t_{0}\right],
\end{gathered}
$$

where $x(t) \in R^{n}$ is the state vector, $\varphi(t)$ is a continuous vector-valued initial function, $A, A_{d}$ are real-valued matrices with appropriate dimensions, $f(\cdot, \cdot): R^{n} \times R \rightarrow R^{n}$ is unknown nonlinear functions satisfying

$$
\|f(x(t), t)\| \leq\|\bar{U} x(t)\|
$$

where $\bar{U}$ is known real constant matrix.

\subsection{Stability Analysis}

Lemma 3.1. Consider system (3.1)-(3.2), for given positive constant $\alpha, \rho_{1}, \rho_{2}, \varepsilon_{1}, \eta_{1}$, if there exist positive definite symmetric matrices $P, S, Q, R$, and any matrices $G, W$ with appropriate dimensions, such that

$$
\left.\begin{array}{ccccccccc}
\multicolumn{10}{c}{Q \leq \rho_{1} I,} & \multicolumn{1}{c}{P \leq \rho_{2} I,} \\
* & G^{T} & W_{d} & A^{T} Q & 0 & d\left(W^{T}+P\right) & A^{T} Q & 0 & \bar{U}^{T} \\
* & -(1-\tau) e^{-\alpha d} R-G-G^{T} & -G & A_{d}^{T} Q & 0 & 0 & 0 & A_{d}^{T} Q & 0 \\
* & * & -d^{-1} e^{-\alpha d} Q & 0 & A_{d}^{T} S & 0 & 0 & 0 & 0 \\
* & * & * & -d^{-1} Q & 0 & 0 & 0 & 0 & 0 \\
* & * & * & * & -d^{2} S & 0 & 0 & 0 & 0 \\
* & * & * & * & * & -S & 0 & 0 & 0 \\
* & * & * & * & * & * & -d^{-1} \varepsilon_{1} Q & 0 & 0 \\
* & * & * & * & * & * & * & -d^{-1} \eta_{1} Q & 0 \\
* & * & * & * & * & * & * & \Phi
\end{array}\right]<0,
$$

holds, then for Lyapunov functional candidate

$$
V(x(t))=x^{T}(t) P x(t)+\int_{t-d(t)}^{t} \dot{x}^{T}(s) e^{-\alpha(t-s)} R \dot{x}(s) d s+\int_{-d}^{0} \int_{t+\theta}^{t} \dot{x}^{T}(r) e^{-\alpha(t-r)} Q \dot{x}(r) d r d \theta,
$$


along the trajectory of system (3.1), there holds the following inequality:

$$
V(x(t))<e^{-\alpha\left(t-t_{0}\right)} V\left(x\left(t_{0}\right)\right)
$$

where $\Sigma=\left(A+A_{d}\right)^{T} P+P\left(A+A_{d}\right)+(\alpha+1) P+R, \Phi=-\left[\tau \rho_{1}\left(1+\varepsilon_{1}+\eta_{1}\right)+\rho_{2}\right]^{-1} I$.

Proof. Let $V_{1}(x(t))=x^{T}(t) P x(t), V_{2}(x(t))=\int_{t-d(t)}^{t} \dot{x}^{T}(s) e^{-\alpha(t-s)} R \dot{x}(s) d s, V_{3}(x(t))=$ $\int_{-d}^{0} \int_{t+\theta}^{t} \dot{x}^{T}(r) e^{-\alpha(t-r)} Q \dot{x}(r) d r d \theta$.

Notice that $x(t)-x(t-d(t))=\int_{t-d(t)}^{t} \dot{x}(r) d r,(3.1)$ can be written as

$$
\dot{x}(t)=\left(A+A_{d}\right) x(t)+f(x(t), t)-A_{d} \int_{t-d(t)}^{t} \dot{x}(r) d r
$$

Along the trajectory of system (3.1), the time derivative of $V_{1}(x(t))$ is given by

$$
\dot{V}_{1}(x(t))=2 x^{T}(t) P\left\{\left(A+A_{d}\right) x(t)+f(x(t), t)\right\}-\int_{t-d(t)}^{t} 2 x^{T}(t) P A_{d} \dot{x}(r) d r .
$$

Let $a=A_{d} \dot{x}(r), b=P x(t)$, from Lemma 2.5 , we can obtain

$$
\begin{aligned}
-2 x^{T}(t) P A_{d} \dot{x}(r) \leq & \dot{x}^{T}(r) A_{d}^{T} X A_{d} \dot{x}(r)+2 \dot{x}^{T}(r) A_{d}^{T} X M P x(t) \\
& +x^{T}(t) P\left(M^{T} X+I\right) X^{-1}(X M+I) P x(t) .
\end{aligned}
$$

Substituting (3.10) into (3.9) leads to

$$
\begin{aligned}
\dot{V}_{1}(x(t)) \leq & x^{T}(t)\left\{\left(A+A_{d}\right)^{T} P+P\left(A+A_{d}\right)+d(t) P\left(M^{T} X+I\right) X^{-1}(M X+I) P\right\} x(t) \\
& +x^{T}(t) P f(x(t), t)+f^{T}(x(t), t) P x(t)+2 x^{T}(t) P M^{T} X A_{d} \int_{t-d(t)}^{t} \dot{x}(r) d r \\
& +\int_{t-d(t)}^{t} \dot{x}^{T}(r) A_{d}^{T} X A_{d} \dot{x}(r) d r \\
\leq & x^{T}(t)\left\{\left(A+A_{d}\right)^{T} P+P\left(A+A_{d}\right)+\tau P\left(M^{T} X+I\right) X^{-1}(X M+I) P\right\} x(t) \\
& +2 x^{T}(t) P f(x(t), t)+2 x^{T}(t) P M^{T} X A_{d} \int_{t-d(t)}^{t} \dot{x}(r) d r+\int_{t-d(t)}^{t} \dot{x}^{T}(r) A_{d}^{T} X A_{d} \dot{x}(r) d r .
\end{aligned}
$$


Differentiating $V_{2}(x(t))$ and $V_{3}(x(t))$ along the trajectory of system (3.1), we have

$$
\begin{aligned}
\dot{V}_{2}(x(t)) \leq & -\alpha \int_{t-d(t)}^{t} e^{-\alpha(t-s)} \dot{x}^{T}(s) R \dot{x}(s) d s+x^{T}(t) R x(t) \\
& -(1-\tau) e^{-\alpha d} x^{T}(t-d(t)) R x(t-d(t)), \\
\dot{V}_{3}(x(t))= & d \dot{x}^{T}(t) Q \dot{x}(t)-\int_{t-d}^{t} \dot{x}^{T}(r) e^{-\alpha(t-r)} Q \dot{x}(r) d r-\alpha \int_{-d}^{0} \int_{t+\theta}^{t} \dot{x}^{T}(r) e^{-\alpha(t-r)} Q \dot{x}(r) d r d \theta \\
\leq & d \dot{x}^{T}(t) Q \dot{x}(t)-e^{-\alpha d} \int_{t-d(t)}^{t} \dot{x}^{T}(r) Q \dot{x}(r) d r-\alpha \int_{-d}^{0} \int_{t+\theta}^{t} \dot{x}^{T}(r) e^{-\alpha(t-r)} Q \dot{x}(r) d r d \theta \\
= & d x^{T}(t) A^{T} Q A x(t)+2 d x^{T}(t-d(t)) A_{d}^{T} Q A x(t)+d x^{T}(t-d(t)) A_{d}^{T} Q A_{d} x(t-d(t)) \\
& -e^{-\alpha d} \int_{t-d(t)}^{t} \dot{x}^{T}(r) Q \dot{x}(r) d r-\alpha \int_{-d}^{0} \int_{t+\theta}^{t} \dot{x}^{T}(r) e^{-\alpha(t-r)} Q \dot{x}(r) d r d \theta \\
& +d\left[2 x^{T}(t) A^{T} Q f(x(t), t)+2 x^{T}(t-d(t)) A_{d}^{T} Q f(x(t), t)+f^{T}(x(t), t) Q f(x(t), t)\right] .
\end{aligned}
$$

By Lemma 2.6, we have

$$
\begin{gathered}
2 x^{T}(t) P f(x(t)) \leq x^{T}(t) P x(t)+f^{T}(x(t)) P f(x(t)), \\
2 x^{T}(t) A^{T} Q f(x(t), t) \leq \varepsilon_{1}^{-1} x^{T}(t) A^{T} Q A x(t)+\varepsilon_{1} f^{T}(x(t), t) Q f(x(t), t), \\
2 x^{T}(t-d(t)) A_{d}^{T} Q f(x(t), t) \leq \eta_{1}^{-1} x^{T}(t-d(t)) A_{d}^{T} Q A_{d} x(t-d(t))+\eta_{1} f^{T}(x(t), t) Q f(x(t), t) .
\end{gathered}
$$

Therefore

$$
\begin{aligned}
\dot{V}(x(t)) & +\alpha V(x(t)) \\
= & \dot{V}_{1}(x(t))+\dot{V}_{2}(x(t))+\alpha V(x(t)) \\
\leq & x^{T}(t)\left\{\left(A+A_{d}\right)^{T} P+P\left(A+A_{d}\right)+d P\left(M^{T} X+I\right) X^{-1}(X M+I) P\right. \\
& \left.\quad+(\alpha+1) P+d\left(1+\varepsilon_{1}^{-1}\right) A^{T} Q A\right\} x(t) \\
& +2 d x^{T}(t-d(t)) A_{d}^{T} Q A x(t)+d\left(1+\eta_{1}^{-1}\right) x^{T}(t-d(t)) A_{d}^{T} Q A_{d} x(t-d(t)) \\
& +2 x^{T}(t) P M^{T} X A_{d} \int_{t-d(t)}^{t} \dot{x}(r) d r \\
& +\int_{t-d(t)}^{t} \dot{x}^{T}(r)\left(A_{d}^{T} X A_{d}-e^{-\alpha d} Q\right) \dot{x}(r) d r+d\left(1+\varepsilon_{1}+\eta_{1}\right) f^{T}(x(t), t) \\
& \times Q f(x(t), t)+f^{T}(x(t)) P f(x(t))
\end{aligned}
$$


Mathematical Problems in Engineering

$$
\begin{aligned}
\leq & x^{T}(t)\left\{\left(A+A_{d}\right)^{T} P+P\left(A+A_{d}\right)+d P\left(M^{T} X+I\right) X^{-1}(X M+I) P\right. \\
& \left.+(\alpha+1) P+d\left(1+\varepsilon_{1}^{-1}\right) A^{T} Q A+d\left(1+\varepsilon_{1}+\eta_{1}\right) \bar{U}^{T} Q \bar{U}+\bar{U}^{T} P \bar{U}\right\} x(t) \\
& +2 d x^{T}(t-d(t)) A_{d}^{T} Q A x(t)+d\left(1+\eta_{1}^{-1}\right) x^{T}(t-d(t)) A_{d}^{T} Q A_{d} x(t-d(t)) \\
& +2 x^{T}(t) P M^{T} X A_{d} \int_{t-d(t)}^{t} \dot{x}(r) d r+\int_{t-d(t)}^{t} \dot{x}^{T}(r)\left(A_{d}^{T} X A_{d}-e^{-\alpha d} Q\right) \dot{x}(r) d r .
\end{aligned}
$$

Notice that $2 x^{T}(t-d(t)) G\left[x(t)-x(t-d(t))-\int_{t-d(t)}^{t} \dot{x}(r) d r\right]=0$, where $G$ is any matrix with appropriate dimension, we have

$$
\dot{V}(x(t))+\alpha V(x(t)) \leq \frac{1}{d(t)} \int_{t-d(t)}^{t} \xi^{T}(t, r) Z \xi(t, r) d r,
$$

where $\xi^{T}(t, r)=\left[x^{T}(t) x^{T}(t-d) \dot{x}^{T}(r)\right]$

$$
Z=\left[\begin{array}{ccc}
\Psi-\bar{U}^{T} \Phi_{1}^{-1} \bar{U} & \tau A^{T} Q A_{d}+G^{T} & d(t) W^{T} A_{d} \\
\tau A_{d}^{T} Q A+G & \tau A_{d}^{T} Q A_{d}-(1-\tau) e^{-\alpha d} R-G-G^{T} & -d(t) G \\
d(t) A_{d}^{T} W & -d(t) G^{T} & d(t)\left(\tau^{-1} A_{d}^{T} S A_{d}-e^{-\alpha \tau} Q\right)
\end{array}\right]
$$

where $\Psi=\left(A+A_{d}\right)^{T} P+P\left(A+A_{d}\right)+d P\left(M^{T} X+I\right) X^{-1}(X M+I) P+(\alpha+1) P+R+d\left(1+\varepsilon_{1}^{-1}\right) A^{T} Q A$. Let $W=X M P, S=\tau X$, by Schur complement lemma, (3.5) is equivalent to the following inequality:

$$
\left[\begin{array}{cccc}
\Psi & d A^{T} Q A_{d}+G^{T} & W^{T} A_{d} & \bar{U}^{T} \\
d A_{d}^{T} Q A+G & d A_{d}^{T} Q A_{d}-(1-\tau) e^{-\alpha d}-G-G^{T} & -G & 0 \\
A_{d}^{T} W & -G^{T} & d^{-2} A_{d}^{T} S A_{d}-d^{-1} e^{-\alpha \tau} Q & 0 \\
\bar{U} & 0 & 0 & \Phi
\end{array}\right]<0 .
$$

Using diag $\{I, I, d(t) I, I\}$ to pre- and post- multiply the left term of (3.17), respectively, we can obtain $Z<0$. Therefore, $\dot{V}(x(t))+\alpha V(x(t))<0$.

The proof is completed. 
Lemma 3.2. Consider system (3.1)-(3.2), for given positive constant $\beta, \rho_{1}, \rho_{2}, \varepsilon_{1}, \eta_{1}$, if there exist positive definite symmetric matrices $P, S, Q$, and any matrices $G, W$ with appropriate dimensions, such that

$$
\left.\begin{array}{ccccccccc}
\multicolumn{10}{c}{Q \leq \rho_{1} I, \quad P \leq \rho_{2} I,} \\
* & G^{T} & W^{T} A_{d} & A^{T} Q & 0 & d\left(W^{T}+P\right) & A^{T} Q & 0 & \bar{U}^{T} \\
* & * & -d^{-1} e^{-\alpha d} Q & 0 & A_{d}^{T} S & 0 & 0 & 0 & 0 \\
* & * & * & -d^{-1} Q & 0 & 0 & 0 & 0 & 0 \\
* & * & * & * & -d^{2} S & 0 & 0 & 0 & 0 \\
* & * & * & * & * & -S & 0 & 0 & 0 \\
* & * & * & * & * & * & -d^{-1} \varepsilon_{1} Q & 0 & 0 \\
* & * & * & * & * & * & * & -d^{-1} \eta_{1} Q & 0 \\
* & * & * & * & * & * & * & * & \Phi
\end{array}\right]<0
$$

holds, then for Lyapunov functional candidate

$$
V(x(t))=x^{T}(t) P x(t)+\int_{-d}^{0} \int_{t+\theta}^{t} \dot{x}^{T}(r) e^{-\alpha(t-r)} Q \dot{x}(r) d r d \theta,
$$

along the trajectory of system (3.1), there holds the following inequality

$$
V(x(t))<e^{\beta\left(t-t_{0}\right)} V\left(x\left(t_{0}\right)\right)
$$

where $\Sigma=\left(A+A_{d}\right)^{T} P+P\left(A+A_{d}\right)-(\beta-1) P$.

Proof. Similarly to the proof line of Lemma 3.1, we can obtain Lemma 3.2.

\subsection{Stabilizing Controller Design}

In this subsection, we will design a stabilizing controller for system (2.12) with delayed switching.

In our design approach we only require the subsystems to be stable during matched period, and the subsystems are allowed to be unstable during mismatched period. Under delayed switching controller $u(t)=K_{\sigma^{\prime}(t)} x(t)$, the corresponding closed-loop system is given by

$$
\begin{gathered}
\dot{x}(t)=\left(A_{\sigma(t)}+B_{\sigma(t)} K_{\sigma^{\prime}(t)}\right) x(t)+A_{d \sigma(t)} x\left(t-d_{\sigma(t)}(t)\right)+f_{\sigma(t)}(x(t), t), \\
x(t)=\varphi(t), \quad t \in\left[t_{0}-d, t_{0}\right] .
\end{gathered}
$$


Let $T^{+}\left(t_{0}, t\right)$ denote the total mismatched period during $\left[t_{0}, t\right), T^{-}\left(t_{0}, t\right)$ denote the total matched period during $\left[t_{0}, t\right)$, then we have the following result.

Theorem 3.3. Consider system (2.12), for given positive constants $\alpha, \beta, \varepsilon_{1}, \eta_{1}, \varepsilon_{2}, \eta_{2}, \rho_{1}, \rho_{2}, \rho_{3}, \rho_{4}$, if there exist positive definite symmetric matrices $X_{i}, Z_{i}, S_{i}, P_{i j}, Q_{i j}, S_{i j}$, and any matrices $Y_{i}, G_{i j}, W_{i j}$ with appropriate dimensions, such that, for $i, j \in \underline{N}, i \neq j$,

$$
\begin{aligned}
& Z_{i} \geq \rho_{1}^{-1} I, \quad X_{i} \geq \rho_{2}^{-1} I, \quad Q_{i j} \leq \rho_{3} I, \quad P_{i j} \leq \rho_{4} I, \\
& {\left[\begin{array}{ccccccccc}
\Pi_{i} & X_{i} & A_{d i} Z_{i} & \Xi_{i} & 0 & 2 d S_{i} & \Xi_{i} & 0 & X_{i} U_{i}^{T} \\
* & -2 Z_{i} & -Z_{i} & Z_{i} A_{d i}^{T} & 0 & 0 & 0 & Z_{i} A_{d i}^{T} & 0 \\
* & * & -d^{-1} e^{-\alpha d} Z_{i} & 0 & Z_{i} A_{d i}^{T} & 0 & 0 & 0 & 0 \\
* & * & * & -d^{-1} Z_{i} & 0 & 0 & 0 & 0 & 0 \\
* & * & * & * & -d^{2} S_{i} & 0 & 0 & 0 & 0 \\
* & * & * & * & * & -S_{i} & 0 & 0 & 0 \\
* & * & * & * & * & * & -d^{-1} \varepsilon_{1} Z_{i} & 0 & 0 \\
* & * & * & * & * & * & * & -d^{-1} \eta_{1} Z_{i} & 0 \\
* & * & * & * & * & * & * & * & \Phi_{1}
\end{array}\right]<0,} \\
& {\left[\begin{array}{ccccccccc}
\Lambda_{i j} & G_{i j}^{T} & W_{i j}^{T} A_{d j} & \Xi_{i j} & 0 & d\left(W_{i j}^{T}+P_{i j}\right) & \Xi_{i j} & 0 & U_{j}^{T} \\
G_{i j} & -G_{i j}-G_{i j}^{T} & -G_{i j} & A_{d j}^{T} Q_{i j} & 0 & 0 & 0 & A_{d j}^{T} Q_{i j} & 0 \\
A_{d j}^{T} W_{i j} & -G_{i j}^{T} & -d^{-1} Q_{i j} & 0 & A_{d j}^{T} S_{i j} & 0 & 0 & 0 & 0 \\
\Xi_{i j}^{T} & Q_{i j} A_{d j} & 0 & -d^{-1} Q_{i j} & 0 & 0 & 0 & 0 & 0 \\
0 & 0 & S_{i j} A_{d j} & 0 & -d^{2} S_{i j} & 0 & 0 & 0 & 0 \\
\tau\left(W_{i j}+P_{i j}\right) & 0 & 0 & 0 & 0 & -S_{i j} & 0 & 0 & 0 \\
\Xi_{i j}^{T} & 0 & 0 & 0 & 0 & 0 & -d^{-1} \varepsilon_{2} Q_{i j} & 0 & 0 \\
0 & Q_{i j} A_{d j} & 0 & 0 & 0 & 0 & 0 & -d^{-1} \eta_{2} Q_{i j} & 0 \\
U_{j} & 0 & 0 & 0 & 0 & 0 & 0 & 0 & \Phi_{2}
\end{array}\right]<0}
\end{aligned}
$$

holds, then under the switching controller $u(t)=K_{\sigma^{\prime}(t)} x(t), K_{i}=Y_{i} X_{i}^{-1}$, and the following average dwell-time scheme:

$$
\inf _{t>t_{0}} \frac{T^{-}\left(t_{0}, t\right)}{T^{+}\left(t_{0}, t\right)} \geq \frac{\beta+\lambda^{*}}{\alpha-\lambda^{*}}, \quad \tau_{a}>\tau_{a}^{*}=\frac{\ln \left(\mu_{1} \mu_{2}\right)}{\lambda^{*}}
$$

the corresponding closed-loop system is exponentially stable, where $0<\lambda^{*}<\alpha, \mu_{1}, \mu_{2} \geq 1$ satisfying $X_{i}^{-1}<\mu_{1} P_{i j}, P_{i j}<\mu_{2} X_{i}^{-1}, Z_{i}^{-1}<\mu_{1} Q_{i j}, Q_{i j}<\mu_{2} Z_{i}^{-1}, \Phi_{1}=-\left[d \rho_{1}\left(1+\varepsilon_{1}+\eta_{1}\right)+\rho_{2}\right]^{-1} I, \Xi_{i}=$ $X_{i} A_{i}^{T}+Y_{i}^{T} B_{i}^{T}, \Pi_{i}=\left(A_{i}+A_{d i}\right) X_{i}+X_{i}\left(A_{i}+A_{d i}\right)^{T}+(\alpha+1) X_{i}+B_{i} Y_{i}+Y_{i}^{T} B_{i}^{T}+R_{i}, \Phi_{2}=$ $-\left[d \rho_{3}\left(1+\varepsilon_{2}+\eta_{2}\right)+\rho_{4}\right]^{-1} I, \Xi_{i j}=\left(A_{j}+B_{j} Y_{i} X_{i}^{-1}\right)^{T} Q_{i j}, \Lambda_{i j}=\left(A_{j}+A_{d j}\right)^{T} P_{i j}+P_{i j}\left(A_{j}+A_{d j}\right)-$ $(\beta-1) P_{i j}+P_{i j} B_{j} Y_{i} X_{i}^{-1}+X_{i}^{-1} Y_{i}^{T} B_{j}^{T} P_{i j}$. 
Proof. Suppose that the $i$ th subsystem is activated at the switching instant $t_{k-1}$, the $j$ th subsystem is activated at the switching instant $t_{k}$, then the corresponding switchings of the controller occur at the switching instant $t_{k-1}+\Delta_{k-1}$ and $t_{k}+\Delta_{k}$, respectively.

When $t \in\left[t_{k-1}+\Delta_{k-1}, t_{k}\right)$, system (3.22) can be written as

$$
\dot{x}(t)=A_{K i} x(t)+A_{d i} x\left(t-d_{i}(t)\right)+f_{i}(x(t), t),
$$

where $A_{K i}=A_{i}+B_{i} K_{i}$.

Consider Lyapunov functional candidate as follows:

$$
V_{i}(x(t))=x^{T}(t) P_{i} x(t)+\int_{t-d(t)}^{t} \dot{x}^{T}(s) e^{-\alpha(t-s)} R_{i} \dot{x}(s) d s+\int_{-d}^{0} \int_{t+\theta}^{t} \dot{x}^{T}(r) e^{-\alpha(t-r)} Q_{i} \dot{x}(r) d r d \theta .
$$

For given positive constants $\alpha, \rho_{1}, \rho_{2}, \varepsilon_{1}, \eta_{1}$, if there exist positive definite symmetric matrices $P_{i}, \widetilde{S}_{i}, Q_{i}, \widetilde{R}_{i}$, and any matrices $G_{i}, W_{i}$ with appropriate dimensions such that the follows matrix inequalities (3.30)-(3.31) hold, then from Lemma 3.1 we have $\dot{V}_{i}(x(t))+$ $\alpha V_{i}(x(t))<0$ :

$$
Q_{i} \leq \rho_{1} I, \quad P_{i} \leq \rho_{2} I,
$$

$$
\left.\begin{array}{ccccccccc}
\Theta_{i} & G_{i}^{T} & W_{i}^{T} A_{d i} & A_{K i}^{T} Q_{i} & 0 & d\left(W_{i}^{T}+P_{i}\right) & A_{K i}^{T} Q_{i} & 0 & U_{i}^{T} \\
* & -(1-\tau) e^{-\alpha d} \widetilde{R}_{i}-G_{i}-G_{i}^{T} & -G_{i} & A_{d i}^{T} Q_{i} & 0 & 0 & 0 & A_{d i}^{T} Q_{i} & 0 \\
* & * & -d^{-1} e^{-\alpha d} Q_{i} & 0 & A_{d i}^{T} \widetilde{S}_{i} & 0 & 0 & 0 & 0 \\
* & * & * & -d^{-1} Q_{i} & 0 & 0 & 0 & 0 & 0 \\
* & * & * & * & -d^{2} \widetilde{S}_{i} & 0 & 0 & 0 & 0 \\
* & * & * & * & * & -\widetilde{S}_{i} & 0 & 0 & 0 \\
* & * & * & * & * & * & -d^{-1} \varepsilon_{1} Q_{i} & 0 & 0 \\
* & * & * & * & * & * & * & -d^{-1} \eta_{1} Q_{i} & 0 \\
* & * & * & * & * & * & * & * & \Phi_{1}
\end{array}\right]
$$

where

$$
\begin{gathered}
\Theta_{i}=\left(A_{K i}+A_{d i}\right)^{T} P_{i}+P_{i}\left(A_{K i}+A_{d i}\right)+(\alpha+1) P_{i}+\widetilde{R}_{i} \\
\Phi_{1}=-\left[d \rho_{1}\left(1+\varepsilon_{1}+\eta_{1}\right)+\rho_{2}\right]^{-1} I .
\end{gathered}
$$

Using diag $\left\{P_{i}^{-1}, Q_{i}^{-1}, Q_{i}^{-1}, Q_{i}^{-1}, S_{i}^{-1}, S_{i}^{-1}, Q_{i}^{-1}, Q_{i}^{-1}, I\right\}$ to pre- and postmultiply the left term of (3.31), and denoting $P_{i}^{-1}=X_{i}, K_{i} P_{i}^{-1}=Y_{i}, Q_{i}^{-1}=Z_{i}, \widetilde{S}_{i}^{-1}=S_{i}, W_{i}=P_{i}, G_{i}=Q_{i}, \widetilde{R}_{i}=0$, we have that (3.31) is equivalent to (3.25). 
Therefore, we have

$$
V_{i}(x(t))<e^{-\alpha\left(t-t_{0}^{i}\right)} V_{i}\left(x\left(t_{0}^{i}\right)\right)
$$

where $t_{0}^{i}$ represents the initial value of the $i$ th subsystem.

When $t \in\left[t_{k}, t_{k}+\Delta_{k}\right)$, system (3.22) can be written as

$$
\dot{x}(t)=A_{K i j} x(t)+A_{d j} x\left(t-d_{j}(t)\right)+f_{j}(x(t), t),
$$

where $A_{K i j}=A_{j}+B_{j} K_{i}$.

Consider Lyapunov functional candidate for system (3.34) as follows:

$$
V_{i j}(x(t))=x^{T}(t) P_{i j} x(t)+\int_{-\tau}^{0} \int_{t+\theta}^{t} \dot{x}^{T}(r) e^{\beta(t-r)} Q_{i j} \dot{x}(r) d r d \theta .
$$

For given positive constants $\beta, \rho_{3}, \rho_{4}, \varepsilon_{2}, \eta_{2}$, if there exist positive definite symmetric matrices $P_{i j}, S_{i j}, Q_{i j}$, and any matrices $G_{i j}, W_{i j}$ with appropriate dimensions such that the follows matrix inequalities (3.36)-(3.37) hold, then from Lemma 3.2 we have $\dot{V}_{i j}(x(t))-\beta V_{i j}(x(t))<0$ :

$$
\begin{aligned}
& Q_{i j} \leq \rho_{3} I, \quad P_{i j} \leq \rho_{4} I, \\
& {\left[\begin{array}{ccccccccr}
\Theta_{i j} & G_{i j}^{T} & W_{i j}^{T} A_{d j} & A_{K i j}^{T} Q_{i j} & 0 & \tau\left(W_{i j}^{T}+P_{i j}\right) & A_{K i j}^{T} Q_{i j} & 0 & U_{j}^{T} \\
G_{i j} & -G_{i j}-G_{i j}^{T} & -G_{i j} & A_{d j}^{T} Q_{i j} & 0 & 0 & 0 & A_{d j}^{T} Q_{i j} & 0 \\
A_{d j}^{T} W_{i j} & -G_{i}^{T} & -\tau^{-1} Q_{i j} & 0 & A_{d j}^{T} S_{i j} & 0 & 0 & 0 & 0 \\
Q_{i j} A_{K i j} & Q_{i j} A_{d j} & 0 & -\tau^{-1} Q_{i j} & 0 & 0 & 0 & 0 & 0 \\
0 & 0 & S_{i j} A_{d j} & 0 & -\tau^{2} S_{i j} & 0 & 0 & 0 & 0 \\
\tau\left(W_{i j}+P_{i j}\right) & 0 & 0 & 0 & 0 & -S_{i j} & 0 & 0 & 0 \\
Q_{i j} A_{K i j} & 0 & 0 & 0 & 0 & 0 & -\tau^{-1} \varepsilon_{2} Q_{i j} & 0 & 0 \\
0 & Q_{i j} A_{d j} & 0 & 0 & 0 & 0 & 0 & -\tau^{-1} \eta_{2} Q_{i j} & 0 \\
U_{j} & 0 & 0 & 0 & 0 & 0 & 0 & 0 & \Phi_{2}
\end{array}\right]<0,}
\end{aligned}
$$

where $\Theta_{i j}=\left(A_{K i j}+A_{d j}\right)^{T} P_{i j}+P_{i j}\left(A_{K i j}+A_{d j}\right)-(\beta-1) P_{i j}, \Phi_{2}=-\left[d \rho_{3}\left(1+\varepsilon_{2}+\eta_{2}\right)+\rho_{4}\right]^{-1} I$.

Denoting $K_{i}=Y_{i} X_{i}^{-1}$, we know that (3.37) is equivalent to (3.26). Thus, we have

$$
V_{i j}(x(t))<e^{\beta\left(t-t_{0}^{i j}\right)} V_{i j}\left(x\left(t_{0}^{i j}\right)\right),
$$

where $t_{0}^{i j}$ represents the initial value of the $j$ th subsystem. 
Let $t_{0}, t_{1}, \ldots, t_{k}$ denote the switching instants in $\left[t_{0}, t\right)$, from (3.33) and (3.38), for $t \geq$ $t_{k}+\Delta_{k}$, we have

$$
\begin{aligned}
V(t) & <e^{-\alpha\left(t-t_{k}-\Delta_{k}\right)} V\left(t_{k}+\Delta_{k}\right) \\
& <\left(\mu_{1} \mu_{2}\right)^{k} e^{-\alpha\left[\left(t-t_{k}-\Delta_{k}\right)+\left(t_{k}-t_{k-1}-\Delta_{k-1}\right)+\cdots+\left(t_{2}-t_{1}-\Delta_{1}\right)+\left(t_{1}-t_{0}-\Delta_{0}\right)\right]+\beta\left(\Delta_{k}+\Delta_{k-1}+\cdots+\Delta_{1}+\Delta_{0}\right)} V\left(t_{0}\right) \\
& =\left(\mu_{1} \mu_{2}\right)^{k} e^{-\alpha T^{-}\left(t_{0}, t\right)+\beta T^{+}\left(t_{0}, t\right)} V\left(t_{0}\right) .
\end{aligned}
$$

By Definition 2.4, we have

$$
k \leq N_{0}+\frac{t-t_{0}}{\tau_{a}} .
$$

From (3.27), it follows that

$$
-T^{-}\left(t_{0}, t\right) \lambda^{-}+T^{+}\left(t_{0}, t\right) \lambda^{+} \leq-\lambda^{*}\left(t-t_{0}\right) .
$$

Substituting (3.40) and (3.41) into (3.39), we have

$$
\begin{aligned}
V(t) & <\left(\mu_{1} \mu_{2}\right)^{N_{0}+\left(t-t_{0}\right) / \tau_{a}} e^{-\lambda^{*}\left(t-t_{0}\right)} V\left(t_{0}\right) \\
& =\left(\mu_{1} \mu_{2}\right)^{N_{0}} e^{\left[\ln \left(\mu_{1} \mu_{2}\right) / \tau_{a}-\lambda^{*}\right]\left(t-t_{0}\right)} V\left(t_{0}\right) .
\end{aligned}
$$

Thus

$$
\|x(t)\|<\sqrt{\frac{b}{a}} \cdot\left(\mu_{1} \mu_{2}\right)^{N_{0} / 2} e^{\left[\ln \left(\mu_{1} \mu_{2}\right) / \tau_{a}-\lambda^{*}\right]\left(t-t_{0}\right) / 2}\left\|x\left(t_{0}\right)\right\|_{h}
$$

where $a=\min _{i, j \in \underline{N}, i \neq j}\left\{\lambda_{\min }\left(X_{i}^{-1}\right), \lambda_{\min }\left(P_{i j}\right)\right\}, b=\max _{i, j \in \underline{N}, i \neq j}\left\{\lambda_{\max }\left(X_{i}^{-1}\right)+\left(\tau^{2} / 2\right) \lambda_{\max }\left(Z_{i}^{-1}\right)\right.$, $\left.\lambda_{\max }\left(P_{i j}\right)+\left(\tau^{2} / 2\right) \lambda_{\max }\left(Q_{i j}\right)\right\}$.

The proof is completed.

Remark 3.4. When $\mu_{1}=\mu_{2}=1$, we have $\tau_{a}^{*}=0$, which implies that switching signals can be arbitrary.

\subsection{Robust Reliable Controller Design}

Now, we are in a position to present sufficient conditions for the existence of robust reliable controller for system (2.1) with delayed switching. 
Theorem 3.5. Consider system (2.1), for given positive constants $\alpha, \beta, \varepsilon_{1}, \eta_{1}, \rho_{1}, \delta_{1}, \varepsilon_{2}, \eta_{2}, \rho_{2}, \delta_{2}$, $\rho_{3}, \delta_{3}, \rho_{4}, \delta_{4}$, if there exist positive definite symmetric matrices $X_{i}, Z_{i}, R_{i}, P_{i j}, Q_{i j}, S_{i j}$, and any matrices $Y_{i}, G_{i j}, W_{i j}$ with appropriate dimensions, such that, for $i, j \in \underline{N}, i \neq j$,

$$
\begin{aligned}
Z_{i} \geq \rho_{1}^{-1} I, \quad X_{i} & \geq \rho_{2}^{-1} I, \quad Q_{i j} \leq \rho_{3} I, \quad P_{i j} \leq \rho_{4} I \\
& {\left[\begin{array}{ccc}
\Omega_{i} & \vartheta_{i} & \Phi_{i} \\
* & \Psi_{i} & 0 \\
* & * & \Gamma_{i}
\end{array}\right]<0, } \\
& {\left[\begin{array}{ccc}
\Omega_{i j} & \vartheta_{i j} & \Phi_{i j} \\
* & \Psi_{i j} & \Delta_{i j} \\
* & * & \Gamma_{i j}
\end{array}\right]<0 }
\end{aligned}
$$

hold, then under the reliable switching controller $u(t)=K_{\sigma /(t)} x(t), K_{i}=Y_{i} X_{i}^{-1}$, and the following average dwell-time scheme:

$$
\inf _{t>t_{0}} \frac{T^{-}\left(t_{0}, t\right)}{T^{+}\left(t_{0}, t\right)} \geq \frac{\beta+\lambda^{*}}{\alpha-\lambda^{*}}, \quad \tau_{a}>\tau_{a}^{*}=\frac{\ln \left(\mu_{1} \mu_{2}\right)}{\lambda^{*}}
$$

the corresponding closed-loop system is exponentially stable,where $0<\lambda^{*}<\alpha, \mu_{1}, \mu_{2} \geq 1$ satisfying $X_{i}^{-1}<\mu_{1} P_{i j}, P_{i j}<\mu_{2} X_{i}^{-1}, Z_{i}^{-1}<\mu_{1} Q_{i j}$

$$
\begin{aligned}
Q_{i j} & <\mu_{2} Z_{i}^{-1}, \quad \Omega_{i}=\left[\begin{array}{ccc}
\Omega_{i 11} & X_{i} & A_{d i} Z_{i} \\
* & -2 Z_{i} & -Z_{i} \\
* & * & -d^{-1} e^{-\alpha d} Z_{i}
\end{array}\right], \\
\Omega_{i 11} & =\left(A_{i}+A_{d i}\right) X_{i}+X_{i}\left(A_{i}+A_{d i}\right)^{T}+(\alpha+1) X_{i}+B_{i} Y_{i}+Y_{i}^{T} B_{i}^{T}+\delta_{1} H_{i} H_{i}^{T}+\delta_{2} B_{i} J_{i} B_{i}^{T}, \\
\vartheta_{i} & =\left[\begin{array}{cccccc}
X_{i} A_{i}^{T}+Y_{i}^{T} B_{i}^{T} & 0 & 2 d R_{i} & X_{i} A_{i}^{T}+Y_{i}^{T} B_{i}^{T} & 0 & X_{i} U_{i}^{T} \\
Z_{i} A_{d i}^{T} & 0 & 0 & 0 & Z_{i} A_{d i}^{T} & 0 \\
0 & Z_{i} A_{d i}^{T} & 0 & 0 & 0 & 0
\end{array}\right], \\
\Phi_{i} & =\left[\begin{array}{cccccc}
X_{i}\left(E_{1 i}+E_{2 i}\right)^{T} & 0 & X_{i} E_{1 i}^{T}+Y_{i}^{T} E_{2 i}^{T} & X_{i} E_{1 i}^{T}+Y_{i}^{T} E_{2 i}^{T} & 0 & Y_{i}^{T} M_{i 0} J_{i}^{1 / 2} \\
0 & Z_{i} E_{2 i}^{T} & Z_{i} E_{2 i}^{T} & 0 & 0 & 0 \\
Z_{i} E_{2 i}^{T} & 0 & 0 & 0 & Z_{i} E_{2 i}^{T} & 0
\end{array}\right],
\end{aligned}
$$




$$
\begin{aligned}
& \Psi_{i}=\operatorname{diag}\left\{-d^{-1} Z_{i}+\delta_{1} H_{i} H_{i}^{T}+\delta_{2} B_{i} J_{i} B_{i}^{T},-d^{2} R_{i}+\delta_{1} H_{i} H_{i}^{T},-R_{i},\right. \\
& \left.-d^{-1} \varepsilon_{1} Z_{i}+\delta_{1} H_{i} H_{i}^{T}+\delta_{2} B_{i} J_{i} B_{i}^{T},-d^{-1} \eta_{1} Z_{i}+\delta_{1} H_{i} H_{i}^{T},-\left[d \rho_{1}\left(1+\varepsilon_{1}+\eta_{1}\right)+\rho_{2}\right]^{-1} I\right\}, \\
& \Gamma_{i}=\operatorname{diag}\left\{-\delta_{1} I,-\delta_{1} I,-\delta_{1} I,-\delta_{1} I,-\delta_{1} I,-\delta_{2} I\right\}, \\
& \Omega_{i j}=\left[\begin{array}{ccc}
\Omega_{i j 1} & G_{i j}^{T} & W_{i j}^{T} A_{d j} \\
* & -G_{i j}-G_{i j}^{T}+2 \delta_{2} E_{2 j}^{T} E_{2 j} & -G_{i j} \\
* & * & -\tau^{-1} Q_{i j}+\delta_{3} E_{2 j}^{T} E_{2 j}
\end{array}\right] \\
& \Omega_{i j 1}=\left(A_{j}+A_{d j}\right)^{T} P_{i j}+P_{i j}\left(A_{j}+A_{d j}\right)-(\beta-1) P_{i j}+P_{i j} B_{j} Y_{i} X_{i}^{-1}+X_{i}^{-1} Y_{i}^{T} B_{j}^{T} P_{i j} \\
& +3 \delta_{3} E_{1 j}^{T} E_{1 j}+\delta_{4} P_{i j} B_{j} J_{i} B_{j}^{T} P_{i j} \\
& \vartheta_{i j}=\left[\begin{array}{cccccc}
\left(A_{j}+B_{j} Y_{i} X_{i}^{-1}\right)^{T} Q_{i j} & 0 & \tau\left(W_{i j}^{T}+P_{i j}\right) & \left(A_{j}+B_{j} Y_{i} X_{i}^{-1}\right)^{T} Q_{i j} & 0 & U_{j}^{T} \\
A_{d j}^{T} Q_{i j} & 0 & 0 & 0 & A_{d j}^{T} Q_{i j} & 0 \\
0 & A_{d j}^{T} S_{i j} & 0 & 0 & 0 & 0
\end{array}\right], \\
& \Phi_{i j}=\left[\begin{array}{ccccc}
P_{i j} H_{j} & 0 & 0 & 0 & W_{i j}^{T} H_{j} \\
0 & 0 & 0 & 0 & 0 \\
0 & 0 & 0 & 0 & 0
\end{array}\right], \quad \Delta_{i j}=\left[\begin{array}{ccccc}
0 & 0 & Q_{i j} H_{j} & 0 & 0 \\
0 & 0 & 0 & 0 & S_{i j} H_{j} \\
0 & 0 & 0 & 0 & 0 \\
0 & 0 & 0 & Q_{i j} H_{j} & 0 \\
0 & Q_{i j} H_{j} & 0 & 0 & 0 \\
0 & 0 & 0 & 0 & 0
\end{array}\right], \\
& \Psi_{i j}=\operatorname{diag}\left\{-d^{-1} Q_{i j}+\delta_{4} Q_{i j} B_{j} J_{i} B_{j}^{T} P_{i j},-d^{2} S_{i j},-S_{i j},-d^{-1} \varepsilon_{2} Q_{i j}+\delta_{4} Q_{i j} B_{j} J_{i} B_{j}^{T} Q_{i j},-d^{-1} \eta_{2} Q_{i j},\right. \\
& \left.-\left[d \rho_{3}\left(1+\varepsilon_{2}+\eta_{2}\right)+\rho_{4}\right]^{-1} I\right\} \\
& \Gamma_{i j}=\operatorname{diag}\left\{-\delta_{3} I,-\delta_{3} I,-\delta_{3} I,-\delta_{3} I,-\delta_{3} I,-\delta_{4} I\right\} .
\end{aligned}
$$


Proof. Consider the following inequalities (3.49) and (3.50):

$$
T_{i}=\left[\begin{array}{ccccccccc}
\widehat{\Pi}_{i} & X_{i} & \widehat{A}_{d i} Z_{i} & \widehat{\Xi}_{i} & 0 & 2 d R_{i} & \widehat{\Xi}_{i} & 0 & X_{i} U_{i}^{T} \\
* & -2 Z_{i} & -Z_{i} & Z_{i} \widehat{A}_{d i}^{T} & 0 & 0 & 0 & Z_{i} \widehat{A}_{d i}^{T} & 0 \\
* & * & -d^{-1} e^{-\alpha d} Z_{i} & 0 & Z_{i} \widehat{A}_{d i}^{T} & 0 & 0 & 0 & 0 \\
* & * & * & -d^{-1} Z_{i} & 0 & 0 & 0 & 0 & 0 \\
* & * & * & * & -d^{2} R_{i} & 0 & 0 & 0 & 0 \\
* & * & * & * & * & * & -d^{-1} \varepsilon_{1} Z_{i} & 0 & 0 \\
* & * & * & * & * & * & * & -d^{-1} \eta_{1} Z_{i} & 0 \\
* & * & * & * & * & * & * & * & \Phi_{1}
\end{array}\right]<0,
$$

$T_{i j}=\left[\begin{array}{ccccccccc}\widehat{\Lambda}_{i j} & G_{i j}^{T} & W_{i j}^{T} \widehat{A}_{d j} & \widehat{\Xi}_{i j} & 0 & d\left(W_{i j}^{T}+P_{i j}\right) & \widehat{\Xi}_{i j} & 0 & U_{j}^{T} \\ * & -G_{i j}-G_{i j}^{T} & -G_{i j} & \widehat{A}_{d j}^{T} Q_{i j} & 0 & 0 & 0 & \widehat{A}_{d j}^{T} Q_{i j} & 0 \\ * & * & -d^{-1} Q_{i j} & 0 & \widehat{A}_{d j}^{T} S_{i j} & 0 & 0 & 0 & 0 \\ * & * & * & -d^{-1} Q_{i j} & 0 & 0 & 0 & 0 & 0 \\ * & * & * & * & -d^{2} S_{i j} & 0 & 0 & 0 & 0 \\ * & * & * & * & * & -S_{i j} & 0 & 0 & 0 \\ * & * & * & * & * & * & * & -d^{-1} \eta_{2} Q_{i j} & 0 \\ * & * & * & * & * & * & * & * & * \\ * & * & * & * & -d^{-1} \varepsilon_{2} Q_{i j} & 0 & 0 \\ \Phi_{2}\end{array}\right]<0$,

where $\Phi_{1}=-\left[d \rho_{1}\left(1+\varepsilon_{1}+\eta_{1}\right)\right]^{-1} I, \widehat{\Xi}_{i}=X_{i} \widehat{A}_{i}^{T}+Y_{i}^{T} M_{i} B_{i}^{T}, \widehat{\Pi}_{i}=\left(\widehat{A}_{i}+\widehat{A}_{d i}\right) X_{i}+X_{i}\left(\widehat{A}_{i}+\widehat{A}_{d i}\right)^{T}+$ $(\alpha+1) X_{i}+B_{i} M_{i} Y_{i}+Y_{i}^{T} M_{i} B_{i}^{T}, \Phi_{2}=-\left[d \rho_{2}\left(1+\varepsilon_{2}+\eta_{2}\right)\right]^{-1} I, \widehat{\Xi}_{i j}=\left(\widehat{A}_{j}+B_{j} M_{i} Y_{i} X_{i}^{-1}\right)^{T} Q_{i j}, \widehat{\Lambda}_{i j}=$ $\left(\widehat{A}_{j}+\widehat{A}_{d j}\right)^{T} P_{i j}+P_{i j}\left(\widehat{A}_{j}+\widehat{A}_{d j}\right)-(\beta-1) P_{i j}+P_{i j} B_{j} M_{i} Y_{i} X_{i}^{-1}+X_{i}^{-1} Y_{i}^{T} M_{i} B_{j}^{T} P_{i j}$.

Substituting (2.3) and (2.10) into (3.49), we can obtain

$$
T_{i}=T_{1 i}+T_{2 i},
$$


where

$$
T_{1 i}=\left[\begin{array}{ccccccccc}
\Pi_{i} & X_{i} & A_{d i} Z_{i} & \Xi_{i} & 0 & 2 d R_{i} & \Xi_{i} & 0 & X_{i} U_{i}^{T} \\
* & -2 Z_{i} & -Z_{i} & Z_{i} A_{d i}^{T} & 0 & 0 & 0 & Z_{i} A_{d i}^{T} & 0 \\
* & * & -d^{-1} e^{-\alpha d} Z_{i} & 0 & Z_{i} A_{d i}^{T} & 0 & 0 & 0 & 0 \\
* & * & * & -d^{-1} Z_{i} & 0 & 0 & 0 & 0 & 0 \\
* & * & * & * & -d^{2} R_{i} & 0 & 0 & 0 & 0 \\
* & * & * & * & * & -R_{i} & 0 & 0 & 0 \\
* & * & * & * & * & * & * & -d^{-1} \eta_{1} Z_{i} & 0 \\
* & * & * & * & * & * & * & * & \Phi_{1}
\end{array}\right],
$$

$$
T_{2 i}=\left[\begin{array}{ccccccccc}
\Upsilon_{i} & 0 & \kappa_{i} & \phi_{i}^{T} & 0 & 0 & \phi_{i}^{T} & 0 & 0 \\
* & 0 & 0 & \kappa_{i}^{T} & 0 & 0 & 0 & \kappa_{i}^{T} & 0 \\
* & * & 0 & 0 & \kappa_{i}^{T} & 0 & 0 & 0 & 0 \\
* & * & * & 0 & 0 & 0 & 0 & 0 & 0 \\
* & * & * & * & 0 & 0 & 0 & 0 & 0 \\
* & * & * & * & * & 0 & 0 & 0 & 0 \\
* & * & * & * & * & * & 0 & 0 & 0 \\
* & * & * & * & * & * & * & 0 & 0 \\
* & * & * & * & * & * & * & * & 0
\end{array}\right],
$$

where $\Pi_{i}=\left(A_{i}+A_{d i}\right) X_{i}+X_{i}\left(A_{i}+A_{d i}\right)^{T}+(\alpha+1) X_{i}+B_{i} M_{i 0} \Upsilon_{i}+Y_{i}^{T} M_{i 0} B_{i}^{T}, \Upsilon_{i}=H_{i} F_{i}\left(E_{1 i}+E_{2 i}\right) X_{i}+$ $X_{i}\left(E_{1 i}+E_{2 i}\right)^{T} F_{i}^{T} H_{i}^{T}+B_{i} M_{i 0} L_{i} Y_{i}+Y_{i}^{T} L_{i} M_{i 0} B_{i}^{T}, \kappa_{i}=H_{i} F_{i} E_{2 i} Z_{i}, \phi_{i}=H_{i} F_{i} E_{1 i} X_{i}+B_{i} M_{i 0} L_{i} Y_{i}$

By Lemma 2.7, Lemma 2.8, and Schur complement lemma, we know that (3.49) is equivalent to (3.45). Similarly, substituting (2.3) into (3.50), we can obtain that (3.50) is equivalent to (3.46).

From Theorem 3.3, we know that Theorem 3.5 holds.

The proof is completed.

Remark 3.6. In actual operation, the condition (3.27) or (3.47) is difficult to check. Let $\Delta_{\max }$ be a known positive scalar that represents the maximum delayed period, a simple condition of the average dwell time is proposed, that is to say, (3.27) or (3.47) can be reduced to the following condition:

$$
\tau_{a}>\tau_{a}^{*}=\max \left\{\frac{\ln \left(\mu_{1} \mu_{2}\right)}{\lambda^{*}},\left(\frac{\beta+\lambda^{*}}{\alpha-\lambda^{*}}+1\right) \Delta_{\max }\right\}, \quad 0<\lambda^{*}<\alpha .
$$


Proof of (3.54): According to (3.27), we have

$$
\begin{aligned}
\frac{T^{-}\left(t_{0}, t\right)}{T^{+}\left(t_{0}, t\right)} & \geq \frac{\beta+\lambda^{*}}{\alpha-\lambda^{*}} \Longrightarrow \frac{\left(t-t_{0}\right)-T^{+}\left(t_{0}, t\right)}{T^{+}\left(t_{0}, t\right)} \geq \frac{\beta+\lambda^{*}}{\alpha-\lambda^{*}} \\
& \Longrightarrow t-t_{0} \geq \frac{\beta+\lambda^{*}}{\alpha-\lambda^{*}} T^{+}\left(t_{0}, t\right)+T^{+}\left(t_{0}, t\right) \\
& \Longrightarrow t-t_{0} \geq\left(\frac{\beta+\lambda^{*}}{\alpha-\lambda^{*}}+1\right) T^{+}\left(t_{0}, t\right) .
\end{aligned}
$$

On the other hand

$$
\begin{aligned}
\left(\frac{\beta+\lambda^{*}}{\alpha-\lambda^{*}}+1\right) T^{+}\left(t_{0}, t\right) & \leq\left(\frac{\beta+\lambda^{*}}{\alpha-\lambda^{*}}+1\right) N_{\sigma(t)}\left(t_{0}, t\right) \Delta_{\max } \\
& \leq\left(\frac{\beta+\lambda^{*}}{\alpha-\lambda^{*}}+1\right) \frac{t-t_{0}}{\tau_{a}^{*}} \Delta_{\max } .
\end{aligned}
$$

Obviously, if the following inequality (3.57) is satisfied, then we have that (3.55) holds

$$
t-t_{0} \geq\left(\frac{\beta+\lambda^{*}}{\alpha-\lambda^{*}}+1\right) \frac{t-t_{0}}{\tau_{a}^{*}} \Delta_{\max }
$$

From (3.57), we have $\tau_{a}^{*} \geq\left(\left(\beta+\lambda^{*}\right) /\left(\alpha-\lambda^{*}\right)+1\right) \Delta_{\max }$.

The proof is completed.

\section{Conclusion}

In this paper, we have investigated the robust reliable stabilization problem for switched nonlinear systems with time-varying delays and delayed switching. The average dwell-time approach is utilized for stability analysis and controller design. Our future work will focus on extending the proposed design method to $H_{\infty}$ performance analysis for switched nonlinear time-varying systems with delayed switching.

\section{Acknowledgments}

This work was supported by the National Natural Science Foundation of China under Grant no. 60974027. The authors would like to thank anonymous reviewers for their valuable comments that greatly improved the quality and presentation of this paper. 


\section{References}

[1] W. Wong and R. W. Brockett, "Systems with finite communication bandwidth constraints. I. State estimation problems," IEEE Transactions on Automatic Control, vol. 42, no. 9, pp. 1294-1299, 1997.

[2] C. Tomlin, G. J. Pappas, and S. Sastry, "Conflict resolution for air traffic management: a study in multiagent hybrid systems," IEEE Transactions on Automatic Control, vol. 43, no. 4, pp. 509-521, 1998.

[3] P. Varaiya, "Smart cars on smart roads: problems of control," IEEE Transactions on Automatic Control, vol. 38, no. 2, pp. 195-207, 1993.

[4] H. Lin and P. J. Antsaklis, "Stability and stabilizability of switched linear systems: a survey of recent results," IEEE Transactions on Automatic Control, vol. 54, no. 2, pp. 308-322, 2009.

[5] Z. Sun and S. S. Ge, "Analysis and synthesis of switched linear control systems," Automatica, vol. 41, no. 2, pp. 181-195, 2005.

[6] J. P. Hespanha, “Uniform stability of switched linear systems: extensions of LaSalle's invariance principle," IEEE Transactions on Automatic Control, vol. 49, no. 4, pp. 470-482, 2004.

[7] R. Wang and J. Zhao, "Guaranteed cost control for a class of uncertain switched delay systems: an average dwell-time method," Cybernetics and Systems, vol. 38, no. 1, pp. 105-122, 2007.

[8] C. De Persis, R. De Santis, and A. S. Morse, "Switched nonlinear systems with state-dependent dwelltime," Systems \& Control Letters, vol. 50, no. 4, pp. 291-302, 2003.

[9] J. Liu, X. Liu, and W.-C. Xie, "Delay-dependent robust control for uncertain switched systems with time-delay," Nonlinear Analysis: Hybrid Systems, vol. 2, no. 1, pp. 81-95, 2008.

[10] M. S. Mohamad, S. Alwan, and X. Z. Liu, "On stability of linear and weakly nonlinear switched systems with time delay," Mathematical and Computer Modelling, vol. 48, no. 7-8, pp. 1150-1157, 2008.

[11] P. Niamsup, "Controllability approach to $H_{\infty}$ control problem of linear time-varying switched systems," Nonlinear Analysis: Hybrid Systems, vol. 2, no. 3, pp. 875-886, 2008.

[12] M. S. Mahmoud, "Delay-dependent $H_{\infty}$ filtering of a class of switched discrete-time state delay systems," Signal Processing, vol. 88, no. 11, pp. 2709-2719, 2008.

[13] Q.-K. Li, J. Zhao, and G. M. Dimirovski, "Tracking control for switched time-varying delays systems with stabilizable and unstabilizable subsystems," Nonlinear Analysis: Hybrid Systems, vol. 3, no. 2, pp. 133-142, 2009.

[14] Y. Zhang, X. Liu, and X. Shen, "Stability of switched systems with time delay," Nonlinear Analysis: Hybrid Systems, vol. 1, no. 1, pp. 44-58, 2007.

[15] Y. G. Sun, L. Wang, and G. M. Xie, "Delay-dependent robust stability and stabilization for discrete-time switched systems with mode-dependent time-varying delays," Applied Mathematics and Computation, vol. 180, no. 2, pp. 428-435, 2006.

[16] C.-H. Lien, K.-W. Yu, Y.-F. Lin, Y.-J. Chung, and L.-Y. Chung, “Robust reliable $H_{\infty}$ control for uncertain nonlinear systems via LMI approach," Applied Mathematics and Computation, vol. 198, no. 1, pp. 453$462,2008$.

[17] B. Yao and F. Wang, "LMI approach to reliable $H_{\infty}$ control of linear systems," Journal of Systems Engineering and Electronics, vol. 17, no. 2, pp. 381-386, 2006.

[18] Y. Liu, J. Wang, and G.-H. Yang, "Reliable control of uncertain nonlinear systems," Automatica, vol. 34, no. 7, pp. 875-879, 1998.

[19] L. Yu, "An LMI approach to reliable guaranteed cost control of discrete-time systems with actuator failure," Applied Mathematics and Computation, vol. 162, no. 3, pp. 1325-1331, 2005.

[20] R. Wang, M. Liu, and J. Zhao, "Reliable $H_{\infty}$ control for a class of switched nonlinear systems with actuator failures," Nonlinear Analysis: Hybrid Systems, vol. 1, no. 3, pp. 317-325, 2007.

[21] R. Wang and J. Zhao, "Robust adaptive control for a class of uncertain switched delay systems with actuator failures," Dynamics of Continuous, Discrete \& Impulsive Systems. Series B, vol. 15, no. 5, pp. 635-645, 2008.

[22] Z. Xiang and R. Wang, "Robust $L_{\infty}$ reliable control for uncertain nonlinear switched systems with time delay," Applied Mathematics and Computation, vol. 210, no. 1, pp. 202-210, 2009.

[23] W. Xie, C. Wen, and Z. Li, "Input-to-state stabilization of switched nonlinear systems," IEEE Transactions on Automatic Control, vol. 46, no. 7, pp. 1111-1116, 2001.

[24] Z. Ji, X. Guo, S. Xu, and L. Wang, "Stabilization of switched linear systems with time-varying delay in switching occurrence detection," Circuits, Systems, and Signal Processing, vol. 26, no. 3, pp. 361-377, 2007.

[25] Z. R. Xiang and R. H. Wang, "Robust control for uncertain switched non-linear systems with time delay under asynchronous switching," IET Control Theory \& Applications, vol. 3, no. 8, pp. 1041-1050, 2009 . 
[26] Z. R. Xiang and R. H. Wang, "Robust stabilization of switched non-linear systems with time-varying delays under asynchronous switching," Proceedings of the Institution of Mechanical Engineers-part I: Journal of Systems and Control Engineering, vol. 223, no. 8, pp. 1111-1128, 2009.

[27] D. Liberzon, Switching in Systems and Control, Systems \& Control: Foundations \& Applications, Birkhäuser, Boston, Mass, USA, 2003.

[28] Y. S. Moon, P. Park, W. H. Kwon, and Y. S. Lee, "Delay-dependent robust stabilization of uncertain state-delayed systems," International Journal of Control, vol. 74, no. 14, pp. 1447-1455, 2001.

[29] Y.-Y. Cao, Y.-X. Sun, and C. Cheng, "Delay-dependent robust stabilization of uncertain systems with multiple state delays," IEEE Transactions on Automatic Control, vol. 43, no. 11, pp. 1608-1612, 1998.

[30] L. Xie, "Output feedback $H_{\infty}$ control of systems with parameter uncertainty," International Journal of Control, vol. 63, no. 4, pp. 741-750, 1996.

[31] I. R. Petersen, "A stabilization algorithm for a class of uncertain linear systems," Systems $\mathcal{E}$ Control Letters, vol. 8, no. 4, pp. 351-357, 1987. 


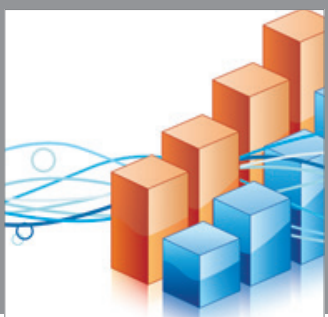

Advances in

Operations Research

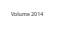

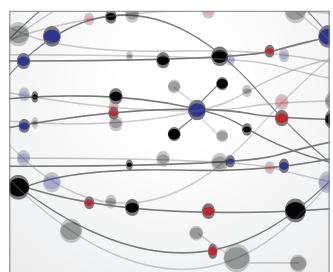

\section{The Scientific} World Journal
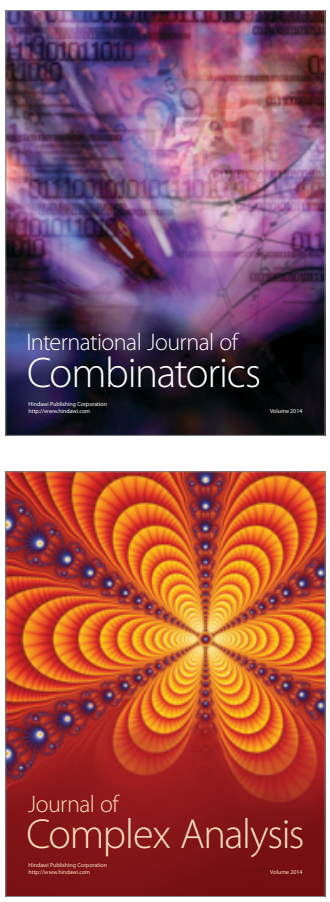

International Journal of

Mathematics and

Mathematical

Sciences
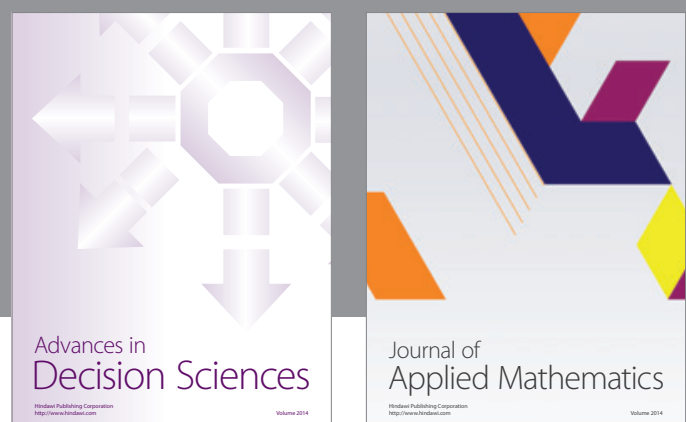

Journal of

Applied Mathematics
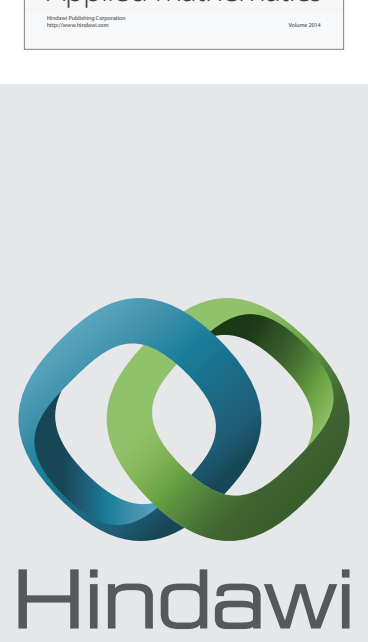

Submit your manuscripts at http://www.hindawi.com
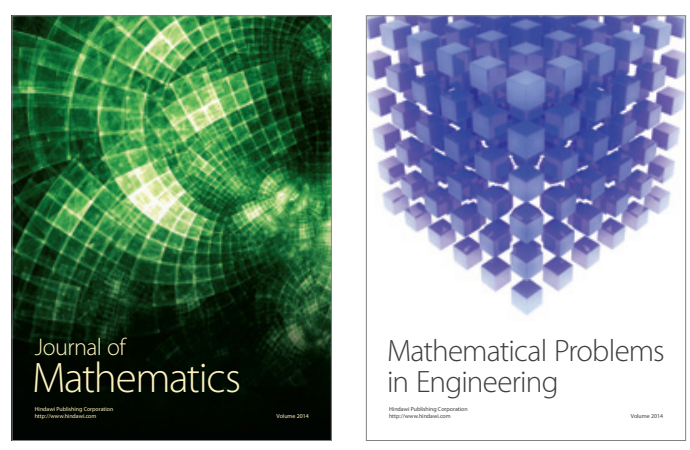

Mathematical Problems in Engineering
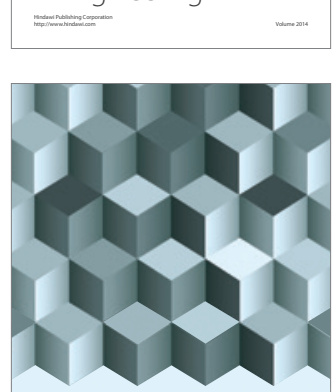

Journal of

Function Spaces
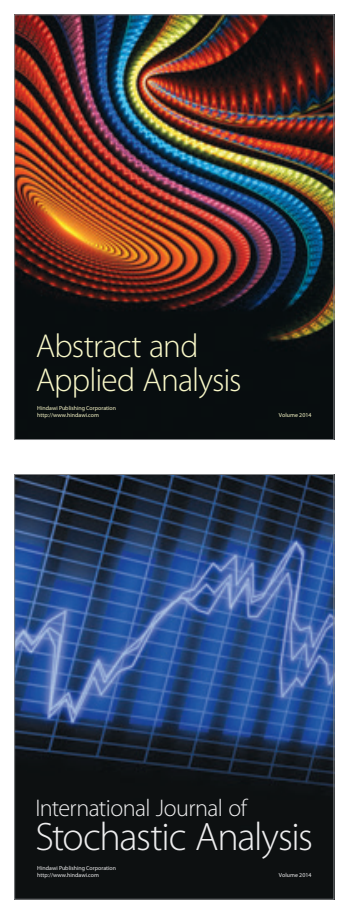

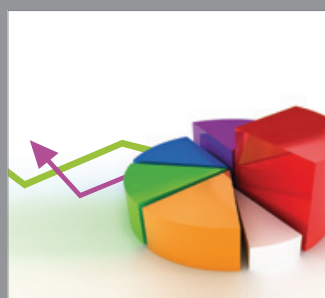

ournal of

Probability and Statistics

Promensencen
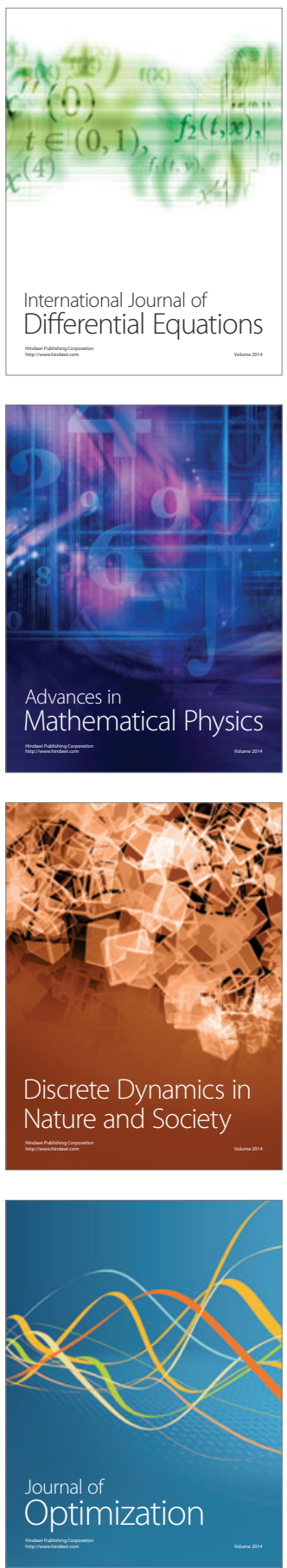